\title{
Genomic selection improves the possibility of applying multiple breeding programs in different environments
}

\author{
M. Slagboom, ${ }^{1 *}$ M. Kargo, ${ }^{1,2}$ A. C. Sørensen, ${ }^{1}$ J. R. Thomasen, ${ }^{1,3}$ and H. A. Mulder ${ }^{4}$ \\ ${ }^{1}$ Department of Molecular Biology and Genetics, Center for Quantitative Genetics and Genomics, Aarhus University, 8830 Tjele, Denmark \\ ${ }^{2}$ SEGES Cattle, 8200 Aarhus N, Denmark \\ ${ }^{3}$ VikingGenetics, 8960 Assentoft, Denmark \\ ${ }^{4}$ Wageningen University \& Research Animal Breeding and Genomics, 6709PB Wageningen, the Netherlands
}

\section{ABSTRACT}

One joint breeding program (BP) for different dairy cattle environments can be advantageous for genetic gain depending on the genetic correlation between environments $\left(r_{g}\right)$. The break-even correlation $\left(r_{b}\right)$ refers to the specific $r_{g}$ where genetic gain with 1 joint $\mathrm{BP}$ is equal to the genetic gain of 2 environment-specific BP. One joint BP has the highest genetic gain if $r_{g}$ is higher than $r_{b}$, whereas 2 environment-specific BP have higher genetic gain if $r_{g}$ is lower than $r_{b}$. Genetic gain in this context is evaluated from a breeding company's perspective that aims to improve genetic gain in both environments. With the implementation of genomic selection, 2 types of collaboration can be identified: exchanging breeding animals and exchanging genomic information. The aim of this study was to study genetic gain in multiple environments with different breeding strategies with genomic selection. The specific aims were (1) to find $r_{b}$ when applying genomic selection; (2) to assess how much genetic gain is lost when applying a suboptimal breeding strategy; (3) to study the effect of the reliability of direct genomic values, number of genotyped animals, and environments of different size on $r_{b}$ and genetic gain; and (4) to find $r_{b}$ from each environment's point of view. Three breeding strategies were simulated: 1 joint $\mathrm{BP}$ for both environments, 2 environment-specific BP with selection of bulls across environments, and 2 environment-specific BP with selection of bulls within environments. The $r_{b}$ was 0.65 and not different from $r_{b}$ with progeny-testing breeding programs when compared at the same selection intensity. The maximum loss in genetic gain in a suboptimal breeding strategy was $24 \%$. A higher direct genomic value reliability and an increased number of genotyped selection candidates increased genetic gain, and the effect on $r_{b}$ was not large. A different size in 2 envi-

Received November 2, 2018.

Accepted May 12, 2019.

*Corresponding author: margotslagboom@mbg.au.dk ronments decreased $r_{b}$ by, at most, 0.10 points. From a large environment's point of view, 1 joint BP was the optimal breeding strategy in most scenarios. From a small environment's point of view, 1 joint BP was only the optimal breeding strategy at high $r_{g}$. When the exchange of breeding animals between environments was restricted, genetic gain could still increase in each environment. This was due to the exchange of genomic information between environments, even when $r_{g}$ between environments were as low as 0.4. Thus, genomic selection improves the possibility of applying environment-specific BP.

Key words: genetic gain, dairy cow, breeding strategy, genotype by environment interaction

\section{INTRODUCTION}

There is evidence that genotype by environment interaction exists in dairy cattle (Nauta et al., 2006; Sundberg et al., 2010; International Bull Evaluation Service, 2014a,b), causing the genetic correlation between environments $\left(\boldsymbol{r}_{\boldsymbol{g}}\right)$ to be $<1$. Collaboration between breeding programs (BP) for different environments can be advantageous for genetic gain but only if $r_{g}$ is high enough. James (1961) suggested that selecting on an index for genetic gain in 2 environments is better than separate selection in both environments when $r_{g}$ is higher than approximately 0.7 . The break-even correlation, as defined by Mulder et al. (2006), refers to the specific $r_{g}$ in which genetic gain in 1 joint $\mathrm{BP}$ with a common breeding goal for both environments is equal to genetic gain in $2 \mathrm{BP}$ each with an environment-specific breeding goal. One joint $\mathrm{BP}$ has the highest genetic gain if $r_{g}$ is higher than the break-even correlation, whereas 2 environment-specific BP have higher genetic gain if $r_{g}$ is lower than the break-even correlation. The driving force behind genetic gain in 1 joint $\mathrm{BP}$ is a higher selection intensity but a lower accuracy of selection, whereas in 2 environment-specific $\mathrm{BP}$, the intensity of selection is lower but the accuracy is higher. Genetic gain in this context is evaluated from 
a breeding company's perspective that aims to improve genetic gain in both environments. The break-even correlation estimated by Mulder et al. (2006) was 0.61 for a classical progeny-testing scheme in dairy cattle.

The implementation of genomic selection might have changed the break-even correlation. With progeny testing, the exchange of breeding animals is linked to the exchange of information used to estimate breeding values. With the implementation of genomic selection, it is possible to split these 2 ways of collaboration. The association between genotypes and phenotypes estimated for one environment can be valuable for another environment, even if no breeding animals are selected across environments. One example of this is that genotypes from progeny-tested bulls from the Norwegian Red breed are exchanged with the Nordic Red breed, which increases accuracies of genomic prediction (Zhou et al., 2014). Genotype by environment interaction might decrease the accuracy of information used across environments, but provided that $r_{g}$ is not too low, reference populations can be used efficiently across environments. In this situation, it might be possible to apply environment-specific BP in small populations by utilizing reference populations from larger populations.

Because genomic selection is widely applied today, it is important to study the break-even correlation with BP that apply genomic selection. The overall aim of this study was to study genetic gain in multiple environments with different breeding strategies with genomic selection. The specific aims were (1) to find the breakeven correlation when applying genomic selection; (2) to assess how much genetic gain is lost when applying a suboptimal breeding strategy; (3) to study the effect of the reliability of direct genomic values, number of genotyped animals, and different sized environments on the break-even correlation and genetic gain; and (4) to find the break-even correlation from each environment's point of view.

\section{MATERIALS AND METHODS}

\section{Breeding Objective}

In this study, we evaluated genetic gain from the point of view of a breeding company that has a market share in 2 environments, similar to the set-up by Mulder et al. (2006). The aim of the breeding company was to improve genetic gain in an objective that covers both environments (equation [1]):

$$
\Delta G_{b c}=w_{1} \Delta G_{1}+w_{2} \Delta G_{2},
$$

where $\Delta G_{b c}=$ genetic gain per year in the objective for the breeding company, $w_{i}=$ relative importance of environment $i\left(w_{1}+w_{2}=1\right)$, and $\Delta G_{i}=$ genetic gain per year in environment $i(i=1,2)$.

The relative importance reflected the size of each environment; $w_{1}$ was the number of cows in the breeding nucleus in environment 1 relative to the total number of cows in both environments. Genetic gain in environment $i$ was represented by genetic gain in the total merit index (TMI) in environment $i$. The TMI was simulated as one trait for simplicity reasons. The TMI in environment 1 and TMI in environment 2 were simulated as separate traits. The genetic parameters of these traits were based on milk production for Holstein cattle in Denmark. The heritability and genetic standard deviation were assumed to be the same in both environments.

\section{Breeding Strategies}

Three breeding strategies were simulated using stochastic simulation (Table 1 and Figure 1): 1 breeding program for both environments with selection of bulls across environments (1bp); 2 breeding programs with selection of bulls across environments (2bp_across); and 2 breeding programs with selection of bulls within environments (2bp_within).

The simulated population resembled the breeding nucleus of a genomic selection scheme for dairy cattle in Denmark. Two environments were simulated in all breeding strategies, each containing 10,000 cows of 1 to $5 \mathrm{yr}$ of age (Figure 1). Every year, 1,000 bull calves and 1,000 heifers were selected for genotyping in each environment, based on parent average. Bull calves coming from embryo transfer were marked in the simulation and were all selected for genotyping. Every year, 50 bulls were selected to be mated to all cows in one environment. These bulls could be selected from all bulls that were genotyped in the current or previous year and were thus 1 or $2 \mathrm{yr}$ of age. Out of the genotyped heifers, 100 were selected to be donors for embryo transfer, and each donor was mated 5 times to different sires, resulting in 2 offspring from each mating. The rest of the cows in the breeding nucleus produced one offspring each. Approximately 1,000 replacement heifers originate from the donors selected for embryo transfer. In breeding strategies $1 \mathrm{bp}$ and 2bp_across, these bulls could be selected across environments. In breeding strategy 2bp_within, bulls could be selected within environment only. In all breeding strategies, cows were selected within environment. Each year, unselected bulls, bulls $>4$ yr of age, cows $>5$ yr of age, and $15 \%$ of randomly selected animals were culled. In breeding strategy $1 \mathrm{bp}$, animals were selected for the TMI in both environments. In breeding strategies 2bp_across and 2bp_within, each environment had 
Table 1. Overview of breeding strategies

\begin{tabular}{llcc}
\hline & & \multicolumn{2}{c}{$\begin{array}{c}\text { Breeding goal }^{1} \\
\text { (economic weights })\end{array}$} \\
\cline { 3 - 4 } Breeding strategy & Selection of bulls & EW1 & EW2 \\
\hline 1bp & Across environments & $w_{1}$ & $w_{2}$ \\
2bp_across & Across environments & 1 & 0 \\
& Within environment & & 1 \\
& & 1 & 0 \\
\hline
\end{tabular}

${ }^{1} \mathrm{EW} 1$ = economic weight of total merit index in environment $1 ; \mathrm{EW} 2=$ economic weight of total merit index in environment $2 ; w_{i}=$ relative importance of environment $i\left(i=1,2 ; w_{1}+w_{2}=1\right) ; w_{1}$ was 0.5 with the basic input parameters.

${ }^{2}$ The first line represents the breeding goal in environment 1 ; the second line represents the breeding goal in environment 2 .

an environment-specific breeding goal (Table 1). Note that the breeding goal in each environment was not the same as $\Delta G_{b c}$. Selection decisions were based on breeding values predicted using the DMU package (Madsen and Jensen, 2013). The procedure used resembled a single-step genomic BLUP; that is, all information was combined in one step.

\section{Simulation}

Each breeding strategy was simulated for $30 \mathrm{yr}$ and replicated 20 times in the stochastic simulation program ADAM (Pedersen et al., 2009). In ADAM, a population of animals was simulated and genetic and phenotypic levels of different traits reported. A pseudo-genomic set-up was used, based on the method developed by Dekkers (2007), which means that no genetic markers or QTL were simulated (see Buch et al. (2012) for a more detailed explanation of this method in ADAM). Instead, 2 genomic traits were simulated that reflected the direct genomic values (DGV) of the TMI in environment 1 and environment 2. For instance, the genetic correlation between the TMI in environment 1 and the DGV of the TMI in environment 1 was equal to the accuracy (square root of the reliability) of the DGV for the TMI in environment 1 . The accuracy of the DGV was constant over years with the basic input parameters, assuming a constant size of the reference population. Thus, no reference population was simulated but the accuracy of the DGV was varied to reflect a change in the size of the reference population.

\section{Input Parameters}

The 3 breeding strategies were simulated with the basic input parameters and with a range of alternative input parameters (Table 2). The genetic correlation between the TMI in environment 1 and the TMI in environment 2 was assumed to be known without error and varied between 0 and 1 , in steps of 0.1 . When the DGV reliability was the same in the 2 environments, scenarios were designated as "rel_same" followed by the reliability in both environments. When the DGV reliability was different in the 2 environments, scenarios were designated as "rel_diff" followed by the reliability in the environment with the lower reliability, because the environment with the higher reliability always had a reliability of 0.65 in rel_diff scenarios. In addition, all

Table 2. Basic input parameters and alternative ranges

\begin{tabular}{lll}
\hline Parameter & Basic input parameters & Alternative range \\
\hline Genetic correlation & $0-1$ & \\
Heritability & 0.44 & $0.67^{2}$ \\
Relative importance of environment $1\left(w_{1}\right)^{1}$ & 0.50 & $0.20,0.45,0.55,0.75,0.90$ \\
Direct genomic value reliability & 0.65 & $250,500,2,000$, or 4,000 per environment \\
Number of genotyped bull calves & 1,000 per environment & \\
Number of selected donors for embryo transfer & 100 per environment & \\
Number of selected bulls & 50 per environment & \\
Number of sires each donor is mated to & 5 per donor & 20,000 per environment $^{2}$ \\
Number of offspring per donor per mating & 2 per mating \\
Number of cows in breeding nucleus & 10,000 per environment & \\
\hline
\end{tabular}

\footnotetext{
${ }^{1} w_{1}+w_{2}=1$.
}

${ }^{2}$ The alternative values for $w_{1}$ and the number of cows in the breeding nucleus were only simulated in the scenarios with 1 small and 1 large environment.

${ }^{3}$ The number of genotyped heifers was equal to the number of genotyped bull calves per year. 
3 breeding strategies were run with input parameters that resembled a small and a large environment. The DGV reliability was fixed at 0.65 in the large environment (environment 1). In the small environment (environment 2), DGV reliability varied from $0.20,0.45$, 0.55 , and 0.65 to simulate a change in the size of the reference population for this environment. No higher DGV reliabilities were simulated in the small environment, because this figure reflects the number of animals in the reference population and we considered it unrealistic that the reference population was larger in the small environment than in the large environment. In the large environment, the number of genotyped bull calves and the number of cows in the breeding nucleus was either the same as in the small environment (basic input parameters) or 2 times the number in the small environment (2,000 genotyped bull calves and 20,000 cows in the breeding nucleus). When the number of genotyped animals was 2,000 and the number of cows in the breeding nucleus was $20,000, \Delta G_{b c}$ (equation [1]) was calculated using a value for $w_{1}$ of 0.67 $\left(w_{1}=\frac{20,000}{20,000+10,000}=0.67\right)$.

\section{Break-Even Correlation}

The genetic level in the last $10 \mathrm{yr}$ of the simulation was used to calculate $\Delta G_{b c}$ for each of the 3 breeding strategies. The specific $r_{g}$ above which it was better for $\Delta G_{b c}$ to apply 1 joint breeding program for both environments than to apply 2 environment-specific BP was the break-even correlation for genetic gain in the breeding company's objective $\left(\boldsymbol{r}_{b, b c}\right)$. Genetic gain per year was plotted for breeding strategies $1 \mathrm{bp}$ and

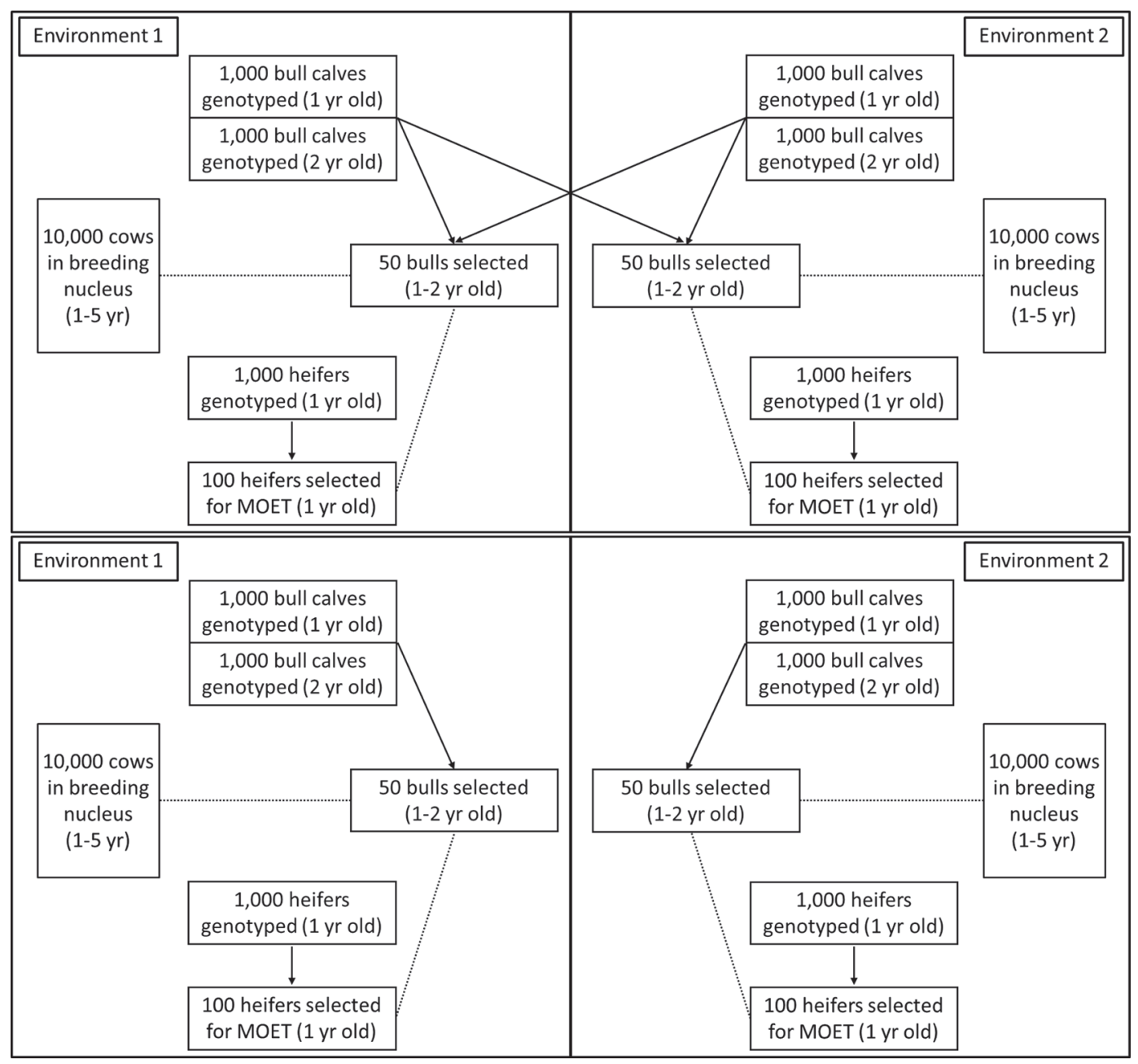

Figure 1. A schematic overview of the breeding schemes simulated in this study. Arrows indicate that a proportion of animals were selected, and dotted lines indicate that groups of animals were mated. The top panels represent the breeding scheme in strategies $1 \mathrm{bp}$ and $2 \mathrm{bp} \_$across; the bottom panels represent the breeding scheme in strategy $2 \mathrm{bp}$ _within. Breeding strategy $1 \mathrm{bp}=1$ breeding program for both environments with selection of bulls across environments, breeding strategy $2 \mathrm{bp}$-across $=2$ breeding programs with selection of bulls across environments, and breeding strategy 2 bp_within $=2$ breeding programs with selection of bulls within environments. MOET $=$ multiple-ovulation embryo transfer. 
2bp_within for $r_{g}$ between 0 and 1 . A linear equation was fitted through each of these lines. The lines were equated (equation [2]) and by rearranging the terms, the intersection of these lines was calculated (equation $[3])$ :

$$
\begin{gathered}
\alpha_{2 b p_{\_} \text {within }}+\beta_{2 b p_{-} \text {within }} \times r_{g}=\alpha_{1 b p}+\beta_{1 b p} \times r_{g}, \\
r_{b, b c}=\frac{\alpha_{2 b p \_ \text {within }}-\alpha_{1 b p}}{\beta_{1 b p}-\beta_{2 b p_{-} \text {within }}}
\end{gathered}
$$

where $r_{g}=$ genetic correlation between environments, $r_{b, b c}=$ break-even correlation for $\Delta G_{b c}, \alpha_{j}=$ intercept of the regression of $\Delta G_{b c}$ in breeding strategy $j$ on $r_{g}$ ( $j=1 \mathrm{bp}, 2 \mathrm{bp} \_$within $)$, and $\beta_{j}=$ regression coefficient of the regression of $\Delta G_{b c}$ in breeding strategy $j$ on $r_{g}$. Genetic gain in breeding strategy 2bp_across was not included because genetic gain in this breeding strategy was not expected to be linear due to the increase in genetic gain from the selection of breeding bulls across environments at $r_{q}>0.80$ (Mulder and Bijma, 2006).

Similarly, break-even correlations were calculated using genetic gain in the TMI for each environment separately; for example, using genetic gain per year in the TMI for environment $1\left(\Delta G_{1}\right)$ for the break-even correlation for $\Delta G_{1}\left(r_{b, 1}\right)$ and genetic gain per year in the TMI for environment $2\left(\Delta G_{2}\right)$ for the break-even correlation for $\Delta G_{2}\left(\boldsymbol{r}_{b, 2}\right)$. These break-even correlations were calculated to determine at which genetic correlation it would be beneficial to have 1 joint breeding program from one environment's point of view. If environments do not benefit at all from each other, genetic gain in breeding strategy $2 \mathrm{bp} \_$within would not increase with an increasing genetic correlation between environments; that is, plotted genetic gain would be a straight line. Therefore, the break-even correlation was also calculated using $\Delta G_{b c}$ in breeding strategy $1 \mathrm{bp}$ and a straight line $\left(\boldsymbol{r}_{b, s l}\right)$. For the straight line, $\alpha_{j}=\Delta G_{b c}$ in breeding strategy 2bp_within at an $r_{g}$ of 0 and $\beta_{j}=0$. All versions of the break-even correlation $\left(r_{b, b c}, r_{b, 1}, r_{b, 2}\right.$, and $r_{b, s l}$ ) were calculated for each of the 20 replicates to find the standard deviation of the break-even correlation, as well as the average of these estimates. The standard deviation of the break-even correlation from the simulation is the standard error of $\widehat{r_{b, b c}}$.

\section{Loss in Genetic Gain in a Suboptimal Breeding Strategy}

To assess how much genetic gain was lost when applying a suboptimal breeding strategy, loss in $\Delta G_{b c}$ was calculated relative to the breeding strategy with the highest $\Delta G_{b c}$ below and above the break-even correlation for the basic input parameters (equation [4]):

$$
G_{\text {loss }}=\left(1-\frac{\Delta G_{-}}{\Delta G_{+}}\right) \times-100 \%,
$$

where $G_{\text {loss }}=$ loss in $\Delta G_{b c}$ in a suboptimal breeding strategy (in \%), $\Delta G_{-}=\Delta G_{b c}$ in a suboptimal breeding strategy, and $\Delta G_{+}=\Delta G_{b c}$ in the optimal breeding strategy; that is, with the highest $\Delta G_{b c}$. $G_{\text {loss }}$ was calculated for every $r_{g}$ from 0 to 1 , in steps of 0.1 . Maximum loss in genetic gain was defined separately below and above the break-even correlation, relative to whichever breeding strategy had the highest genetic gain below and above the break-even correlation.

\section{Increase in Genetic Gain with Restricted Selection}

We investigated the effect of an increasing $r_{g}$ on genetic gain with the exchange of genomic information but without the exchange of breeding animals across environments. For this, genetic gain in breeding strategy 2bp_within at an $r_{g}$ of 0 was compared with that in the same breeding strategy at an $r_{g}$ of 1 for the basic input parameters (equation [5]):

$$
G_{\text {inc }}=\frac{\Delta G_{2 b p \_ \text {within }, r 1}}{\Delta G_{2 b p \_ \text {within }, r 0}} \times 100 \%,
$$

where $G_{i n c}=$ increase in $\Delta G_{b c}$ in breeding strategy 2bp_within (in \%), $\Delta G_{2 b p_{-} \text {within, } r 1}=\Delta G_{b c}$ in breeding strategy 2bp_within at an $r_{g}$ of 1 , and $\Delta G_{2 b p_{-} \text {within, } r 0}=$ $\Delta G_{b c}$ in breeding strategy 2bp_within at an $\bar{r}_{g}$ of 0 .

\section{Effect of Alternative Input Parameters}

The simulations were run with several alternative input parameters, such as a different number of genotyped bull calves or a different DGV reliability (Table 2 ). To assess the effect of alternative input parameters compared with the basic input parameters, genetic gain was calculated with alternative input parameters relative to genetic gain with the basic input parameters in breeding strategy $1 \mathrm{bp}$ at an $r_{g}$ of 1 (equation [6]):

$$
G_{a l t}=\frac{\Delta G_{1 b p, a l t e r n a t i v e}}{\Delta G_{1 b p, \text { basic }}} \times 100 \%
$$

where $G_{a l t}=$ relative gain in $\Delta G_{b c}$ for alternative input parameters compared with the basic input parameters (in \%), $\Delta G_{1 b p, \text { alternative }}=\Delta G_{b c}$ in breeding strategy $1 \mathrm{bp}$ 


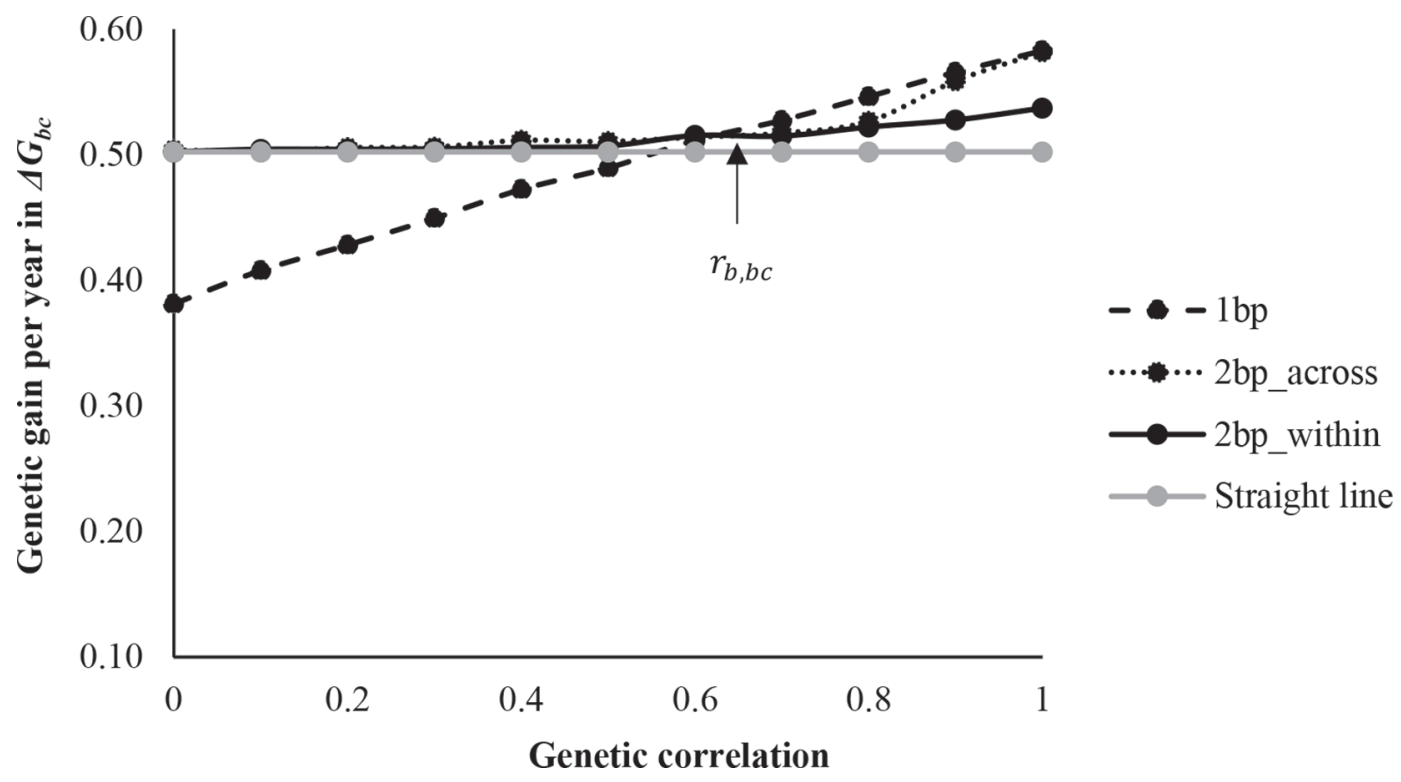

Figure 2. Genetic gain in the breeding goal for the breeding company $\left(\Delta G_{b c}\right)$, for the 3 breeding strategies with the basic input parameters and a straight line, where the intercept $=\Delta G_{b c}$ in breeding strategy 2bp_within at a genetic correlation $\left(r_{q}\right)$ of 0 and slope $=0 . \Delta G_{b c}=w_{1} \Delta G_{1}$ $+w_{2} \Delta G_{2}$, where $w_{i}=$ relative importance of environment $i\left(w_{1}+w_{2}=1\right)$, and $\Delta G_{i}=$ genetic gain per year in environment $i(i=1,2) ; w_{1}$ was 0.5 with the basic input parameters. Breeding strategy $1 \mathrm{bp}=1$ breeding program for both environments with selection of bulls across environments, breeding strategy $2 \mathrm{bp} \_$across $=2$ breeding programs with selection of bulls across environments, and breeding strategy $2 \mathrm{bp} \_$within $=2$ breeding programs with selection of bulls within environments. The arrow indicates $r_{b, b c}$, the break-even correlation for $\Delta G_{b c}$.

at an $r_{g}$ of 1 , with alternative input parameters, $\Delta G_{1 b p, \text { basic }}=\Delta G_{b c}$ in breeding strategy 1 bp at an $r_{g}$ of 1 , with the basic input parameters.

\section{RESULTS}

\section{Break-Even Correlation}

Genetic gain in the breeding objective for the breeding company is shown in Figure 2, for all 3 breeding strategies and a straight line with the same intercept as breeding strategy $2 \mathrm{bp} \_$within. When $r_{g}$ increased, $\Delta G_{b c}$ increased in breeding strategy $1 \mathrm{bp}$. Breeding strategy 2bp_across was equal to breeding strategy $2 \mathrm{bp} \_$within in terms of $\Delta G_{b c}$ until environments were genetically highly correlated $\left(r_{g}>0.8\right)$. At that point, bulls started being selected across environments in breeding strategy 2bp_across. In breeding strategy 2bp_within, selection of bulls across environments was not possible, but there was still an increase in $\Delta G_{b c}$ when environments were more correlated. In Figure 2, the $r_{b, b c}$ can be seen as the intersection of the lines of breeding strategy $1 \mathrm{bp}$ with the lines of breeding strategy 2bp_across and 2bp_within, indicated by an arrow. The break-even correlation calculated using equations [2] and [3], $r_{b, b c}$, was 0.65 (SD 0.02) with the basic input parameters. Breeding strategy $1 \mathrm{bp}$ had the highest $\Delta G_{b c}$ when $r_{g}$ was above the $r_{b, b c}$, and breeding strategies $2 \mathrm{bp} \_$across and 2bp_within had higher $\Delta G_{b c}$ when $r_{g}$ was below the $r_{b, b c}$.

\section{Maximum Loss in Genetic Gain}

Below the $r_{b, b c}$, applying $1 \mathrm{BP}$ compared with $2 \mathrm{BP}$ for both environments caused a loss in genetic gain, $G_{\text {loss }}$ (equation [4]). Maximum $G_{\text {loss }}$ below the $r_{b, b c}$ of 0.65 was found at an $r_{g}$ of 0 : breeding strategy $1 \mathrm{bp}$ caused $24.3 \%$ loss in $\Delta G_{b c}$ compared with breeding strategy 2bp_across (Table 3). Above the break-even correlation, applying $2 \mathrm{BP}$ compared with $1 \mathrm{BP}$ for both environments caused a loss in genetic gain. Maximum $G_{\text {loss }}$ above the $r_{b, b c}$ was found when applying breeding strategy $2 \mathrm{bp} \_$within; $G_{\text {loss }}$ was $7.8 \%$ compared with applying breeding strategy $1 \mathrm{bp}$ at a $r_{g}$ of 1 . Maximum $G_{\text {loss }}$ above the $r_{b, b c}$ when applying breeding strategy $2 \mathrm{bp}$ _across compared with $1 \mathrm{bp}$ was $3.6 \%$, at an $r_{g}$ of 0.8. Maximum $G_{\text {loss }}$ when applying a suboptimal breeding strategy below the $r_{b, b c}$ was far greater than that above the $r_{b, b c}$.

\section{Value of Genomic Information Sharing}

In breeding strategies 2bp_across and 2bp_within, $\Delta G_{b c}$ increased with an increasing $r_{g}$ from 0 to 1 . In breeding strategy 2bp_across, both breeding animals and genomic information could be exchanged between 
environments, whereas in breeding strategy 2bp_within, only the exchange of genomic information could increase $\Delta G_{b c}$. This increase can be seen when comparing genetic gain in breeding strategy $2 \mathrm{bp} \_$within with the straight line in Figure 2. This increase, $G_{i n c}$ (equation [5]), was $7 \%$ at an $r_{g}$ of 1 compared with genetic gain at an $r_{g}$ of 0 . Calculating the break-even correlation with a straight line resulted in an $r_{b, s l}$ of 0.57 (SD 0.04), lower than the $r_{b, b c}$ of 0.65 . Thus, sharing genomic information between environments increased the break-even correlation and, in correlated environments, genetic gain increased even when breeding animals were not exchanged between environments.

\section{Effect of Alternative Input Parameters}

$D G V$ Reliability. A lower DGV reliability for the trait in both environments decreased $\Delta G_{b c}$. In the scenario where the DGV reliability was $0.20, G_{\text {alt }}$ (equation [6]) was $76.1 \%$ compared with the basic situation when the DGV reliability was 0.65 (Table 4). Increasing the DGV reliability from 0.65 to 0.90 increased genetic gain by $6.9 \%$. A lower and a higher DGV reliability slightly increased $r_{b, b c}$ compared with the basic input parameters, and the standard deviation of $r_{b, b c}$ decreased with a higher DGV reliability. The largest difference found in $r_{b, b c}$ for the range of reliabilities we tested was 0.04; thus, the effect of the DGV reliability on the breakeven correlation was not large.
Number of Genotyped Selection Candidates. When the number of genotyped selection candidates was reduced to 250 per environment, $G_{\text {alt }}$ was $91.7 \%$ (Table 4). Increasing the number of genotyped selection candidates from 1,000 to 4,000 only slightly increased $G_{\text {alt }}$. An increased number of genotyped selection candidates increased the selection intensity and increased $r_{b, b c}$. The effect on $r_{b, b c}$ was clear when increasing from 250 to 500 , but this effect was less pronounced when increasing the number of selection candidates further. The largest difference found in $r_{b, b c}$ for the range of number of genotyped selected candidates we tested was 0.06 . Thus, increasing the number of genotyped selection candidates primarily affected $r_{b, b c}$ and genetic gain in the overall objective when the initial number of genotyped selection candidates was low.

Different Size of Environments. The effect of different sizes of environments and a different DGV reliability in environment 2 (rel_diff scenarios) on $G_{\text {alt }}$ and $r_{b, b c}$ are shown in Table 5 . When environment 1 was larger than environment $2, \Delta G_{b c}$ was calculated with $w_{1}=0.67$. The scenario with the basic input parameters had the highest $\Delta G_{b c}$ in breeding strategy 1 bp (equal sized and a DGV reliability of 0.65 in both environments). When environments were of different sizes, $r_{b, b c}$ was smaller than when environments were the same size. A higher DGV reliability in environment 2 increased $r_{b, b c}$. This effect was more pronounced when environments were of different sizes; $r_{b, b c}$ increased by 0.05 points when the DGV reliability increased from

Table 3. Genetic gain per year in the breeding goal for the breeding company $\left(\Delta G_{b c}{ }^{1}\right)$, and loss in $\Delta G_{b c}\left(G_{l o s s}{ }^{2}\right)$ in a suboptimal breeding strategy in percentages below or above the break-even correlation for $\Delta G_{b c}\left(r_{b, b c}\right)$ for the 3 breeding strategies ${ }^{3}$ with the basic input parameters

\begin{tabular}{|c|c|c|c|c|c|c|}
\hline \multirow[b]{3}{*}{ Genetic correlation } & \multicolumn{6}{|c|}{ Breeding strategy } \\
\hline & \multicolumn{2}{|c|}{$1 \mathrm{bp}$} & \multicolumn{2}{|c|}{ 2bp_across } & \multicolumn{2}{|c|}{ 2bp_within } \\
\hline & $\Delta G_{b c}$ & $G_{\text {loss }}(\%)$ & $\Delta G_{b c}$ & $G_{\text {loss }}(\%)$ & $\Delta G_{b c}$ & $G_{\text {loss }}(\%)$ \\
\hline 0.0 & 0.38 & -24.3 & 0.50 & 0.0 & 0.50 & -0.3 \\
\hline 0.2 & 0.43 & -15.3 & 0.51 & 0.0 & 0.50 & -0.2 \\
\hline 0.4 & 0.47 & -7.6 & 0.51 & 0.0 & 0.51 & -1.0 \\
\hline 0.6 & 0.51 & -0.5 & 0.51 & 0.0 & 0.52 & 0.2 \\
\hline \multicolumn{7}{|l|}{$r_{b, b c}=0.65$} \\
\hline 0.8 & 0.55 & 0.0 & 0.53 & -3.6 & 0.52 & -4.4 \\
\hline 1.0 & 0.58 & 0.0 & 0.58 & -0.2 & 0.54 & -7.8 \\
\hline
\end{tabular}

${ }^{1} \Delta G_{b c}=w_{1} \Delta G_{1}+w_{2} \Delta G_{2}$, where $w_{i}=$ relative importance of environment $i\left(w_{1}+w_{2}=1\right)$, and $\Delta G_{i}=$ genetic gain per year in environment $i(i=1,2)$. $w_{1}$ was 0.5 with the basic input parameters.

${ }^{2} G_{\text {loss }}=\left(1-\frac{\Delta G_{-}}{\Delta G_{+}}\right) \times-100 \%$, where $\Delta G_{-}=\Delta G_{b c}$ in a suboptimal breeding strategy and $\Delta G_{+}=\Delta G_{b c}$ in the most optimal breeding strategy. The most optimal breeding strategy below $r_{b, b c}$ was 2 bp_across and the most optimal breeding strategy above $r_{b, b c}$ was $1 \mathrm{bp}$.

${ }^{3}$ Breeding strategy $1 \mathrm{bp}=1$ breeding program for both environments with selection of bulls across environments, breeding strategy $2 \mathrm{bp} \_$across $=2$ breeding programs with selection of bulls across environments, and breeding strategy $2 \mathrm{bp} \_$within $=2$ breeding programs with selection of bulls within environments. 
Table 4. Effect of reliability of direct genomic values (DGV) and number of genotyped selection candidates on the break-even correlation for $\Delta G_{b c}{ }^{1}\left(r_{b, b c}{ }^{2}\right)$ and relative genetic gain in $\Delta G_{b c}$ for alternative input parameters compared with the basic input parameters in percentages $\left(G_{a l t}^{3}\right)$

\begin{tabular}{llccc}
\hline Number genotyped & DGV reliability & $r_{b, b c}$ & SD of $r_{b, b c}$ & $G_{\text {alt }}(\%)$ \\
\hline 1,000 & 0.20 & 0.69 & 0.05 & 76.1 \\
& 0.45 & 0.66 & 0.03 & 91.4 \\
& 0.55 & 0.65 & 0.03 & 95.5 \\
& 0.65 & 0.65 & 0.02 & 100.0 \\
& 0.75 & 0.66 & 0.02 & 102.7 \\
250 & 0.90 & 0.69 & 0.02 & 106.9 \\
500 & 0.65 & 0.61 & 0.03 & 91.7 \\
1,000 & & 0.64 & 0.02 & 96.7 \\
2,000 & & 0.65 & 0.02 & 100.0 \\
4,000 & & 0.66 & 0.02 & 100.3 \\
\hline
\end{tabular}

${ }^{1} \Delta G_{b c}=$ genetic gain per year in the objective for the breeding company.

${ }^{2} r_{b, b c}=\frac{\alpha_{2 b p_{\_} \text {within }}-\alpha_{1 b p}}{\beta_{1 b p}-\beta_{2 b p \_ \text {within }}}$, where $\alpha_{j}=$ intercept of the regression of $\Delta G_{b c}$ in breeding strategy $j$ on $r_{g}(j=1 \mathrm{bp}$,

2bp_within; $1 \mathrm{bp}=1$ breeding program for both environments with selection of bulls across environments, $2 \mathrm{bp} \_$within $=2$ breeding programs with selection of bulls within environments), $\beta_{j}=$ regression coefficient of the regression of $\Delta G_{b c}$ in breeding strategy $j$ on $r_{g}$.

${ }^{3} G_{\text {alt }}=\frac{\Delta G_{1 b p, \text { alternative }}}{\Delta G_{1 b p, \text { basic }}} \times 100 \%$, where $\Delta G_{1 b p, \text { alternative }}=\Delta G_{b c}$ in breeding strategy 1 bp at an $r_{g}$ of 1 , with alternative input parameters, $\Delta G_{1 b p, b a s i c}=\Delta G_{b c}$ in breeding strategy 1 bp at an $r_{q}$ of 1 , with the basic input parameters (DGV reliability of 0.65 and number of genotyped selection candidates of 1,000 ).

0.20 to 0.65 . Thus, $r_{b, b c}$ was lower and the effect of different reliabilities in 2 environments on $r_{b, b c}$ was more pronounced when environments were of different size.

\section{Break-Even Correlation from Each Environment's Point Of View}

Different Size of Environments. In Figure 3, $\Delta G_{b c}, \Delta G_{1}$, and $\Delta G_{2}$ are shown for $r_{g}$ of 0 to 1 for scenario rel_diff 0.45 , with environments of different size. From the large environment's point of view, breeding strategy 1 bp was optimal for $\Delta G_{1}$, regardless of $r_{g}$. This means that the break-even correlation using $\Delta G_{1}\left(r_{b, 1}\right.$, equation [2] and [3]) was 0 in scenario rel_diff 0.45 . For scenarios rel_diff 0.20 and rel_diff $0.55, r_{b, 1}$ was also 0 or negative. In scenario rel_same $0.65, r_{b, 1}$ increased to 0.13 (SD 0.10). Thus, from the large environment's point of view, applying separate BP was not beneficial for most $r_{g}$, in the case of different-sized environments.

From the point of view of environment 2, the small environment, breeding strategy $1 \mathrm{bp}$ was only optimal above the break-even correlation using $\Delta G_{2}, r_{b, 2}$, which was 0.79 (SD 0.02) for scenario rel_diff 0.45 (Figure 3). In Figure $4, r_{b, 2}$ is shown for different sizes of environments and different DGV reliabilities in environment 2. For all values of DGV reliability in environment $2, r_{b, 2}$ was approximately 0.79 with different sizes of environments. This means that from the small environment's point of view, applying 1 breeding program was not beneficial for most $r_{g}$.

Equal Size of Environments. When environments were the same size, break-even correlations from each environment's point of view were more similar to $r_{b, b c}$ than when environments were of different sizes. The lowest $r_{b, 1}$ was 0.54 , in scenario rel_diff 0.20 , and the highest $r_{b, 1}$ was 0.66 in scenario rel_same 0.65 . The different values of the DGV reliability in environment 2 did not affect $r_{b, 2}$ much when environments were the same size (Figure 4).

\section{Effect of DGV Reliability on Genetic Gain in the Small Environment}

In Figure 5, $\Delta G_{2}$ in breeding strategy 2bp_within is shown for $r_{q}$ of 0 to 1 and different input parameters for the DGV reliability. Simulations with environments of a different size are not included because the size of environment 1 did not have an effect on $\Delta G_{2}$ in breeding strategy 2bp_within. However, the effect of a lower DGV reliability on $\Delta G_{2}$ was very pronounced. In the rel_diff scenarios, the decreasing effect of a lower DGV reliability on $\Delta G_{2}$ was present at low $r_{q}$ but diluted at high $r_{g}$. The clearest example of this was the effect on $\Delta G_{2}$ in scenario rel_diff $0.20 ; \Delta G_{2}$ at an $r_{q}$ of 0 was at approximately the same level as in scenario rel_same 0.20 , whereas at an $r_{g}$ of $1, \Delta G_{2}$ was approximately the 
Table 5. Effect of different sizes of environments and reliability of direct genomic values (DGV) in environment 2 on break-even correlation for $\Delta G_{b c}{ }^{1}\left(r_{b, b c}{ }^{2}\right)$ and relative genetic gain in $\Delta G_{b c}$ for alternative input parameters compared with the basic input parameters in percentages $\left(G_{a l t}{ }^{3}\right)$

\begin{tabular}{llccc}
\hline Size of environment & & & & \\
& $\begin{array}{l}\text { DGV reliability in } \\
\text { environment } 2^{5}\end{array}$ & $r_{b, b c}$ & SD of $r_{b, b c}$ & $G_{\text {alt }}(\%)$ \\
\hline Equal size & 0.20 & 0.62 & 0.03 & 93.7 \\
& 0.45 & 0.65 & 0.03 & 96.5 \\
Different size & 0.55 & 0.66 & 0.01 & 98.0 \\
& 0.65 & 0.65 & 0.02 & 100.0 \\
& 0.20 & 0.53 & 0.04 & 92.2 \\
& 0.45 & 0.56 & 0.02 & 94.3 \\
& 0.55 & 0.57 & 0.03 & 95.5 \\
& 0.65 & 0.58 & 0.03 & 97.5 \\
\hline
\end{tabular}

${ }^{1} \Delta G_{b c}=$ genetic gain per year in the objective for the breeding company.

${ }^{2} r_{b, b c}=\frac{\alpha_{2 b p_{-} \text {within }}-\alpha_{1 b p}}{\beta_{1 b p}-\beta_{2 b p \text { within }}}$, where $\alpha_{j}=$ intercept of the regression of $\Delta G_{b c}$ in breeding strategy $j$ on $r_{g}(j=1 \mathrm{bp}$, 2bp_within; $1 \mathrm{bp}=1$ breeding program for both environments with selection of bulls across environments, $2 \mathrm{bp} \_$within $=2$ breeding programs with selection of bulls within environments), $\beta j=$ regression coefficient of the regression of $\Delta G_{b c}$ in breeding strategy $j$ on $r_{g}$.

${ }^{3} G_{\text {alt }}=\frac{\Delta G_{1 b p, \text { alternative }}}{\Delta G_{1 b p, \text { basic }}} \times 100 \%$, where $\Delta G_{1 b p, \text { alternative }}=\Delta G_{b c}$ in breeding strategy 1bp at an $r_{g}$ of 1 , with alternative input parameters, $\Delta G_{1 b p, b a s i c}=\Delta G_{b c}$ in breeding strategy 1 bp at an $r_{g}$ of 1 , with the basic input parameters (DGV reliability of 0.65 and number of genotyped selection candidates of 1,000).

${ }^{4}$ Equal size: input parameters were equal to the basic input parameters for both environments; different size: in environment 1, there were 20,000 cows in the breeding nucleus and 2,000 bull calves and 2,000 heifers were genotyped every year, and input parameters were equal to the basic input parameters in environment 2 . When environments were the same size, a value of $w_{1}=0.5$ was used for the calculation of $\Delta G_{b c}$. When environments were a different size, a value of $w_{1}=0.67$ was used.

${ }^{5}$ The DGV reliability in environment 1 was equal to 0.65 .

level of $\Delta G_{2}$ in scenario rel_same 0.65 (Figure 5). Thus, a lower DGV reliability in one environment had only a small decreasing effect on $\Delta G_{2}$ if $r_{g}$ was high.

\section{DISCUSSION}

\section{Optimal Breeding Strategy}

In this study, different breeding strategies were compared to find the break-even correlation in a breeding scheme with genomic selection. Above an $r_{g}$ of 0.65 , it was more beneficial for $\Delta G_{b c}$ to apply 1 breeding program for 2 environments; below this genetic correlation, it was more beneficial to apply 2 separate BP, one for each environment. The loss in $\Delta G_{b c}$ when applying breeding strategy 2 bp_across was very small, at maximum $4 \%$ at an $r_{g}$ of 0.8 . An increased number of genotyped selection candidates or decreased number of selected breeding bulls (and thus an increased selection intensity) will likely account for this loss in genetic gain. However, the loss in $\Delta G_{b c}$ when applying 1 breeding program for both environments when $r_{g}$ was lower than $r_{b, b c}$ caused a loss in genetic gain of up to $24 \%$. This means that if $r_{g}$ is unknown, implementing an environment-specific breeding program where selection from other environments is allowed is a very beneficial breeding strategy for genetic gain, with a low risk of loss in genetic gain.

\section{Impact of Genomic Selection}

In the basic situation in this study, 50 bulls were selected from 2 age classes with 1,000 genotyped bulls each, from 2 environments (in the breeding strategies where selection across environments was possible). This means a selected proportion of breeding bulls of $1.25 \%$. In Mulder et al. (2006), the number of test-bulls was fixed at 400 and the proportion of sires to breed bulls was $5 \%$ in the basic situation. Comparing the breakeven correlation at the same proportion of selected breeding bulls means looking at the situation in this study where the number of genotyped bull calves per year per environment was 250 (250 per age class per environment, thus from 2 age classes and 2 environments means 1,000 selection candidates). At that number of genotyped bull calves, the break-even correlation was 0.61 , exactly the same as in the progeny-testing scheme used by Mulder et al. (2006). Thus, when comparing at the same selection intensity, the break-even correlation is the same in a progeny-testing breeding scheme and in a breeding scheme applying genomic selection. However, selection intensity generally increases with 

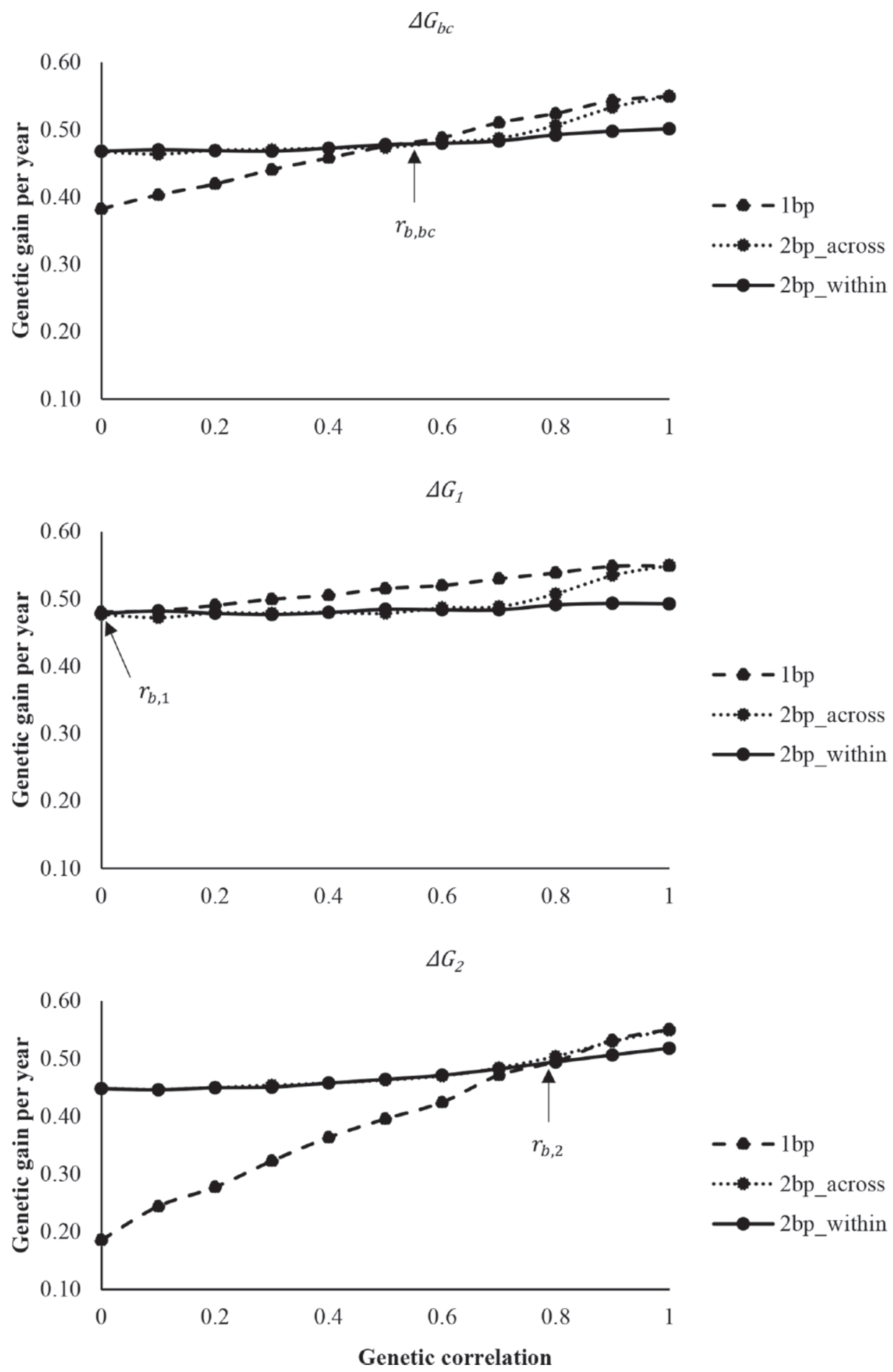

Figure 3. Genetic gain in the breeding goal for the breeding company ( $\Delta G_{b c}$, top panel); the total merit index in the large environment, environment $1\left(\Delta G_{1}\right.$, middle panel); and the total merit index in the small environment, environment $2\left(\Delta G_{2}\right.$, bottom panel), for the 3 breeding strategies in scenario "rel_diff 0.45 " with environments of different size. $\Delta G_{b c}=w_{1} \Delta G_{1}+w_{2} \Delta G_{2}$, where $w_{1}=$ relative importance of environment $i\left(i=1,2 ; w_{1}+w_{2}=1\right)$. The number of genotyped bull calves was 2,000 in environment 1 and 1,000 in environment 2 ; the number of cows in the breeding nucleus was 20,000 in environment 1 and 10,000 in environment 2; the direct genomic values (DGV) reliability was 0.65 in environment 1 and 0.45 in environment 2 ; and $w_{1}$ was 0.67 . Breeding strategy $1 \mathrm{bp}=1$ breeding program for both environments with selection of bulls across environments, breeding strategy 2 bp_across $=2$ breeding programs with selection of bulls across environments, and breeding strategy $2 \mathrm{bp} \_$within $=2$ breeding programs with selection of bulls within environments. The arrows indicate the break-even correlation for $\Delta G_{b c}\left(r_{b, b c}\right)$, the break-even correlation for $\Delta G_{1}\left(r_{b, 1}\right)$, and the break-even correlation for $\Delta G_{2}\left(r_{b, 2}\right)$. 
the implementation of genomic selection, as discussed by Mulder (2007). In that case, genomic selection can increase the break-even correlation but only slightly (from 0.61 to 0.65 as observed in this study).

Simulating breeding schemes with genomic selection instead of a progeny-testing scheme means we can see the effect on genetic gain and the break-even correlation even when selection is restricted to one environment. We showed that genetic gain increases in a correlated environment even when selection of bulls is restricted within each environment, due to the exchange of genomic information between environments. The TMI for both environments become more similar with an increasing $r_{q}$, such that information on the TMI in environment 1 becomes more valuable when breeding for the TMI in environment 2. Essentially, the size of the reference population increases, thereby increasing the DGV accuracy. The increase in genetic gain in breeding strategy 2bp_within was $7 \%$ when $r_{g}$ increased from 0 to 1 . This increase was solely due to an increase in accuracy; without this increase in accuracy, genetic gain in breeding strategy 2bp_within would be a straight line (Figure 2). The break-even correlation calculated with this straight line, $r_{b, s l}$, was 0.08 points lower than $r_{b, b c}$ with the basic input parameters. This indicates that without the increase in accuracy due to genomic

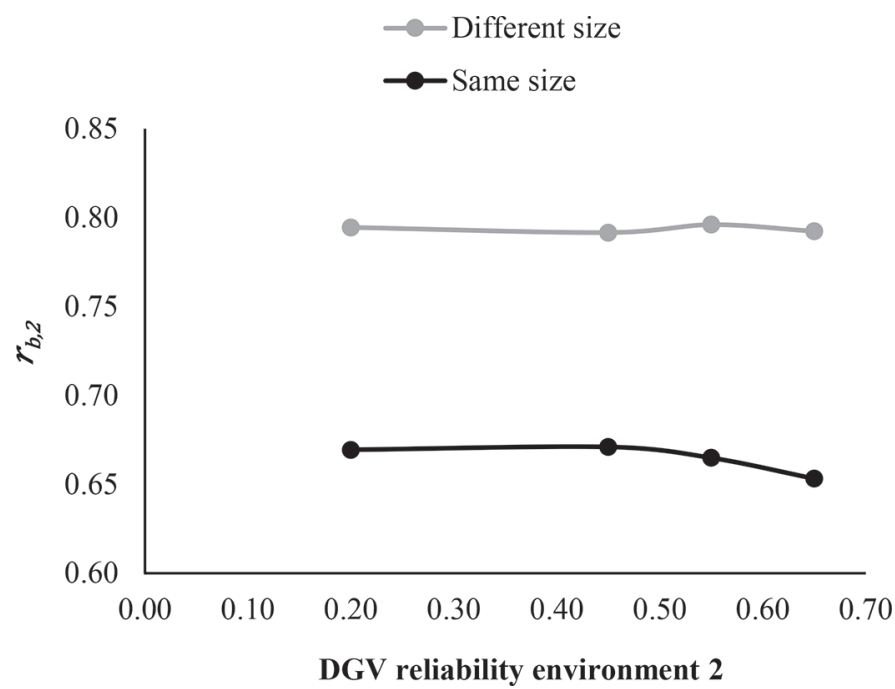

Figure 4. Break-even correlations calculated using genetic gain in the total merit index (TMI) for environment $2\left(r_{b, 2}\right)$ for environments of different size and different direct genomic values (DGV) reliabilities in environment 2. The DGV reliability in environment 1 was equal to 0.65 . When environments were the same size, input parameters were equal to the basic input parameters for both environments, and when environments were a different size, there were 20,000 cows in the breeding nucleus and 2,000 bull calves and 2,000 heifers were genotyped every year in environment 1 ; input parameters were equal to the basic input parameters in environment 2 . information, scenario $1 \mathrm{bp}$ would be optimal for genetic gain at lower $r_{g}$.

How valuable it is to share genomic information depends on the size of the reference population in both the foreign environment and the environment of interest. In the case of a very high DGV accuracy in the large environment and a very low DGV accuracy in the small environment, sharing genomic information can already be beneficial at very low $r_{g}$. In Figure 5, $\Delta G_{2}$ in scenario rel_diff 0.20 starts increasing from $r_{g}=$ 0.4. This indicates that the use of genomic information across environments is very important to help smaller environments with lower DGV reliabilities increase the accuracy of selection and consequently genetic gain. On the condition that populations in different environment are not completely separated genetically, genomic selection can limit the loss in genetic gain in correlated environments due to small reference populations, even when genetic correlations between environments are relatively low.

\section{Break-Even Correlation from Each Environment}

In this study, we also studied genetic gain and the break-even correlation from each environment's point of view. In the rel_diff 0.45 scenario, when environments were of different size, genetic gain in the large environment was highest in breeding strategy $1 \mathrm{bp}$ regardless of $r_{g}$. The economic weight for $\Delta G_{1}$ in the breeding goal in breeding strategy $1 \mathrm{bp}$ was twice that for $\Delta G_{2}$. Hence, there was more emphasis on improving $\Delta G_{1}$ when environments had 1 common breeding program, working in favor of the large environment. This means that $r_{b, 1}$ was very low when environment 1 was larger. When environments were the same size, the economic weights for $\Delta G_{1}$ and $\Delta G_{2}$ were the same, so there was no more emphasis on environment 1 in the breeding goal in breeding strategy $1 \mathrm{bp}$. In this case, $r_{b, 1}$ was not lower than $r_{b, b c}$ with equal reliabilities in both environments. Thus, the effect of increased importance for the large environment in the breeding goal decreased $r_{b, 1}$ dramatically.

For $\Delta G_{2}$, breeding strategy $1 \mathrm{bp}$ was less favorable because of the lower economic weight in the breeding goal when environments were a different size. Therefore, $r_{g}$ needed to be high in order for breeding strategy 1 bp to be favorable for $\Delta G_{2}$. This resulted in a very high $r_{b, 2}$, meaning that from the small environment's point of view, it was only beneficial to collaborate with the large environment if environments were genetically highly correlated.

Studying the break-even correlation from only one environment's point of view is beneficial for research purposes but, in practice, both environments need to 
agree to have one joint breeding program. One environment can always select breeding animals from another environment, but this will be more beneficial if the other environment has the same or a very similar breeding goal. To breed for the same breeding goal, some form of mutual agreement between environments is necessary.

\section{Effect of Alternative Input Parameters}

An increased number of genotyped selection candidates with a constant number of selected breeding animals increased the break-even correlation, comparable to what Mulder et al. (2006) found. They showed that with a decreased selected proportion of breeding animals (and thus a higher selection intensity), the break-even correlation increases. Both an increased and a decreased DGV reliability increased the break-even correlation in this study. However, the effect was so small that we cannot draw firm conclusions. An increased DGV reliability is comparable to an increased number of progeny per bull in a progeny-testing scheme (because both increase the accuracy of selection), which had a small decreasing effect on the break-even correlation in the study by Mulder et al. (2006). In general, the more genetic gain an environment can achieve by itself due to, for instance, an increase in accuracy or selection intensity, the higher the break-even correlation will be.

We only looked at one alternative value of $w_{1}$, when environments were of different size, but this effect was larger than that of different reliabilities in the 2 environments. The main findings from simulating 2 different-sized environments were that these scenarios yielded less genetic gain, $r_{b, b c}$ was lower, and the impact of an increasing reliability on $r_{b, b c}$ in the smaller environment was more pronounced when environments were of different sizes. Mulder et al. (2006) showed that the effect of different values of $w_{1}$ had a larger effect on the break-even correlation than the number of progeny per bull or different values of heritability, which corroborates our findings.

\section{Implications}

The results of this study can be applied to practical dairy cattle BP. For most European countries, $r_{g}$ are very high (International Bull Evaluation Service, 2014a,b); thus, it is very important to look at the higher range of $r_{g}$ when applying the results of this study to practical dairy cattle BP. This means that breeding strategy 1 bp or 2bp_across would be the most beneficial breeding strategy for most practical dairy cattle BP. Interestingly, because $r_{g}$ between European countries and New Zealand for milk production (International Bull Evaluation Service, 2014a) are between the break-even correlation and the genetic correlation point where BP start selection bulls from each other (Smith and Banos, 1991; Mulder and Bijma, 2006), the optimal breeding strategy would be to have $1 \mathrm{BP}$ for both environments. However, these estimates do not include breeding goal

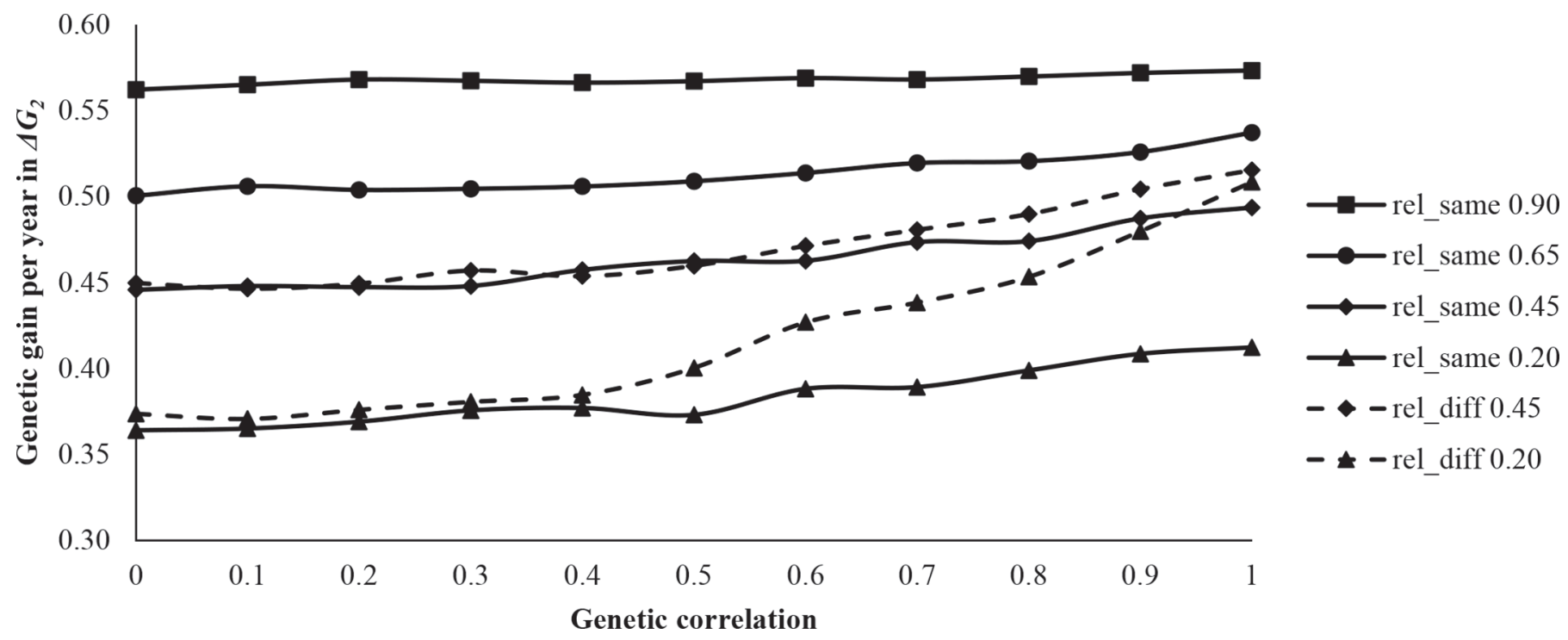

Figure 5. Genetic gain in the total merit index in environment $2\left(\Delta G_{2}\right)$ in breeding strategy 2bp_within for different correlations and different direct genomic values (DGV) reliabilities. "rel_same" indicates that the reliability was the same in both environments and is followed by the number used for the reliability in each scenario; "rel_diff" indicates that the reliability was different in the 2 environments and is followed by the number used for the reliability in environment 2 in each scenario. The reliability in environment 1 was always 0.65 in the rel_diff scenarios. 
differences and thus the $r_{q}$ between TMI will most likely be lower. In this case, breeding strategy 2 bp_across or 2bp_within would yield the highest genetic gain.

The results from breeding strategy 2bp_within are important to demonstrate the effect of exchanging genomic information on genetic gain in correlated environments. A small breed such as the Danish Jersey has a low reliability for using genomic information because of a small reference population (Thomasen et al., 2012). This study shows that without the use of breeding animals across environments, genetic gain can increase by using genomic information from a correlated environment, already at $r_{g}$ as low as 0.4 . If breeding companies are willing to exchange genomic information across environments, increased genetic gain can be achieved in small populations in this way. In addition, such a breeding strategy could be possible in, for example, organic dairy production, where the practice of embryo transfer is not allowed. This could have the consequence that breeding bulls from conventional dairy production cannot be used in an organic environment. In this case, it is important to see how much genetic gain is lost when restricting selection. When this decision is made, it is necessary to assess how to stay competitive in terms of genetic gain; for example, by genotyping more animals. Depending on the situation and environment, breeding strategy 2bp_within can still be a beneficial breeding strategy.

\section{CONCLUSIONS}

When compared at the same selection intensity, the break-even correlation with the implementation of genomic selection is not different from the break-even correlation in a breeding scheme with progeny testing. The maximum loss in genetic gain when applying 2 environment-specific BP compared with one overall BP is minimal when selection of breeding animals across environments is possible, with the input parameters in this study. The application of genomic selection can increase genetic gain in a correlated environment even when breeding animals are not selected across environments, due to the exchange of genomic information. Thus, genomic selection improves the possibility of applying multiple environment-specific BP in different environments.

\section{ACKNOWLEDGMENTS}

The project SOBcows is part of the Organic RDD 2 program, which is coordinated by the International Centre for Research in Organic Food Systems (Tjele,
Denmark). It has received grants from the Green Growth and Development program under the Danish Ministry of Food, Agriculture and Fisheries (Copenhagen).

\section{REFERENCES}

Buch, L. H., M. K. Sørensen, P. Berg, L. D. Pedersen, and A. C. Sørensen. 2012. Genomic selection strategies in dairy cattle: Strong positive interaction between use of genotypic information and intensive use of young bulls on genetic gain. J. Anim. Breed. Genet. 129:138-151. https://doi.org/10.1111/j.1439-0388.2011.00947.x.

Dekkers, J. C. M. 2007. Prediction of response to marker assisted and genomic selection using selection index theory. J. Anim. Breed. Genet. 124:331-341. https://doi.org/10.1111/j.1439-0388.2007 .00701.x.

International Bull Evaluation Service. 2014a. Interbull Routine Genetic Evaluation for Production Traits, April 2014. Accessed July 30, 2018. http://www.interbull.org/web/static/mace_evaluations _archive/eval/prod-apr14.html.

International Bull Evaluation Service. 2014b. Interbull Routine Genetic Evaluation for Udder Health Traits, April 2014. Accessed July 30, 2018. http://www.interbull.org/web/static/mace_evaluations _archive/udder/uder-apr14.html.

James, J. W. 1961. Selection in two environments. Heredity 16:145 152.

Madsen, P., and J. Jensen. 2013. A User's Guide to DMU. Version 6, release 5.2. Aarhus University Foulum, Denmark.

Mulder, H. A. 2007. Methods to optimize livestock breeding programs with genotype by environment interaction and genetic heterogeneity of environmental variance. $\mathrm{PhD}$ thesis. Wageningen University, Wageningen, the Netherlands.

Mulder, H. A., and P. Bijma. 2006. Benefits of cooperation between breeding programs in the presence of genotype by environment interaction. J. Dairy Sci. 89:1727-1739. https://doi.org/10.3168/ jds.S0022-0302(06)72241-X.

Mulder, H. A., R. F. Veerkamp, B. J. Ducro, J. A. M. Van Arendonk, and P. Bijma. 2006. Optimization of dairy cattle breeding programs for different environments with genotype by environment interaction. J. Dairy Sci. 89:1740-1752. https://doi.org/10.3168/ jds.S0022-0302(06)72242-1.

Nauta, W. J., R. F. Veerkamp, E. W. Brascamp, and H. Bovenhuis. 2006. Genotype by environment interaction for milk production traits between organic and conventional dairy cattle production in the Netherlands. J. Dairy Sci. 89:2729-2737. https://doi.org/10 .3168/jds.S0022-0302(06)72349-9.

Pedersen, L. D., A. C. Sørensen, M. Henryon, S. Ansari-Mahyari, and P. Berg. 2009. ADAM: A computer program to simulate selective breeding schemes for animals. Livest. Sci. 121:343-344. https://doi .org/10.1016/j.livsci.2008.06.028.

Smith, C., and G. Banos. 1991. Selection within and across populations in livestock improvement. J. Anim. Sci. 69:2387-2394. https: //doi.org/10.2527/1991.6962387x.

Sundberg, T., L. Rydhmer, W. F. Fikse, B. Berglund, and E. Strandberg. 2010. Genotype by environment interaction of Swedish dairy cows in organic and conventional production systems. Acta Agric. Scand. A Anim. Sci. 60:65-73. https://doi.org/10.1080/09064702 .2010.496003.

Thomasen, J. R., B. Guldbrandtsen, G. Su, R. F. Brøndum, and M. S. Lund. 2012. Reliabilities of genomic estimated breeding values in Danish Jersey. Animal 6:789-796. https://doi.org/10.1017/ S1751731111002035.

Zhou, L., B. Heringstad, G. Su, B. Guldbrandtsen, T. H. E. Meuwissen, M. Svendsen, H. Grove, U. S. Nielsen, and M. S. Lund. 2014. Genomic predictions based on a joint reference population for the Nordic Red cattle breeds. J. Dairy Sci. 97:4485-4496. https://doi .org/10.3168/jds.2013-7580. 